\title{
La Casa Behrens: análisis de una obra de arte total
}

The Behrens House: analysis of a total work of art

\author{
Andrea Isabel Aranda Gómez \\ Departamento de Historia del Arte, Universidad de Málaga, España (drearango@gmail.com)
}

Recibido el 12 de diciembre de 2017; revisado el 10 de enero de 2018; aceptado el 18 de febrero de 2018; publicado el 21 de marzo de 2018

RESUMEN: La Casa Behrens, construida en 1901 por el arquitecto y diseñador Peter Behrens, es uno de los mejores ejemplos de "obra de arte total” en la Historia del Arte. Sin embargo, más allá del logro de una estética total, es decir, la armonía visual y espacial entre cada uno de los elementos que componen la casa, su planificación incluye aspectos que enlazan con la arquitectura moderna del siglo $\mathrm{XX}$, así como con una dimensión social de la obra de arte total que se fue configurando a lo largo del siglo pasado. El análisis de esta obra, basado en el libro que le dedicó el propio Behrens, pretende profundizar en los aspectos propuestos, desentrañando la riqueza teórica y formal que contiene la obra.

PALABRAS ClAVE: Casa Behrens, Obra de arte total, Jugendstil, Colonia de Darmstadt, Arquitectura, Diseño.

\begin{abstract}
The Behrens House was built in 1901 by the architect and designer Peter Behrens and it still being nowadays one of the best examples of "total work of art" in the History of Art. However, beyond the achievement of a total aesthetic, that is, the visual and spatial harmony between each of the elements that make up the house, its planning includes aspects that link with the modern architecture of the 20th century, as well as with a single dimension social of the total work of art that was configured over the past century. The analysis of this work, based on the book that Behrens himself dedicated, seeks to deepen the proposed aspects, unraveling the theoretical and formal wealth contained in the work.
\end{abstract}

KEYWORDS: Behrens House, Gesamtkunstwerk, Jugendstil, Darmstadt Colony, Architecture, Design. 


\section{Contexto: Jugendstil, Gesamtkunstwerk}

Peter Behrens (Alemania, 1868-1940) formó parte de la generación de artistas como Theodor Fischer, Hermann Muthesius, Joseph Maria Olbrich, Henry van de Velde o Richard Riemerschmid, quienes comenzaron su carrera en las últimas décadas del siglo XIX (Kadatz, 1977, pp.5-10). En este contexto fueron fundamentales las influencias del movimiento inglés Arts and Crafts, que, liderado por William Morris, había surgido en Inglaterra alrededor de 1880 como respuesta a la industrialización y sus nuevos métodos de producción. Esta corriente aspiraba a una sociedad en la que el hombre, una vez liberado de su alienación, alcanzase la armonía consigo mismo y su trabajo. Para lograr este propósito, la artesanía debía volver a ejercerse en talleres semejantes a los gremios medievales, dando solución a la problemática de los objetos de mala calidad producidos en masa (Banz y Beierdorf, 2015, p.1).

En Alemania, estas influencias se tradujeron en un deseo por devolver al artista el valor esencial de su trabajo y lograr una reconciliación del arte con la naturaleza. Con este propósito, artistas y artesanos se asociaron en distintas ciudades en torno a talleres llamados "Talleres Unidos por el Arte y la Artesanía" (Vereinigte Werkstätten für Kunst im Handwerk), en los que se rescataron viejas técnicas como la xilografía, llevando también a cabo reformas que aspiraban solucionar de un modo más pragmático el problema que la revolución industrial había evidenciado en Inglaterra. Las distintas medidas tomadas en los talleres se acompañaron de otra serie de reformas que pretendían modernizar la enseñanza en las escuelas de artes y oficios, además de promocionar exposiciones nacionales y regionales, como la de Darmstadt en 1901, con el objetivo de dar a conocer entre el público todas las novedades al respecto (Nerdinger, 2007, p.16).

El Jugendstil ${ }^{1}$ buscó referentes estéticos en la naturaleza y lo exótico, desarrollando un estilo de imágenes inspiradas en el mundo vegetal, animal y humano, con una tendencia a la estilización y la asimetría y un uso ornamental de motivos considerablemente más abstractos si se comparan con los utilizados en estilos coetáneos relacionados, como el Modernismo en España o el Art Nouveau en Francia $^{2}$. La línea, utilizada profusamente en la Casa Behrens, adquiere en esta corriente una entidad

\footnotetext{
${ }^{1}$ El movimiento Jugendstil toma su nombre de Jugend (en español, “Juventud”), una revista semanal sobre arte y artesanía editada por primera vez en Múnich en 1896. La publicación fue vista como la primera gran divulgadora de los diseños creados por los artistas en este nuevo estilo (Dewiel, 2002, pp.5-11).

${ }^{2} \mathrm{Al}$ respecto, se debe tener en cuenta el peso que ejerció en Alemania el deseo por parte de los artistas de romper con la arraigada tradición académica historicista, que basaba la decoración en la aplicación de modelos ornamentales determinados afines a una estética concreta (Kadatz, 1977 p.6). Asimismo, y en relación con esta tendencia hacia motivos abstractos, resulta importante la influencia que el arte y la artesanía japonesa tuvo en el Jugendstil alemán. En este arte, difundido entre los artistas europeos a mediados del siglo XIX, el color y la línea adquieren un gran significado
} 
propia, convirtiéndose en uno de los motivos favoritos del movimiento. Al mismo tiempo, existe otra variante estilística en la que predomina la decoración geométrica, lo que da a lugar la categorización generalista que divide el Jugendstil según su vertiente lineal o geométrica. Junto con Peter Behrens, Henry van de Velde es otro de los mayores representantes del estilo lineal, mientras que Joseph Maria Olbrich o, en Escocia, Charles Rennie Mackintosh lo son del geométrico (Wichmann, 1984).

Desde el campo de la arquitectura, los artistas persiguieron en ocasiones la creación de una “obra de arte total" (Gesamtkunstwerk), un concepto que, a pesar de ser acuñado en el siglo XIX por el compositor Richard Wagner, adquirió una nueva dimensión a principios del XX. Mientras en su origen hacía referencia a la síntesis de las artes, es decir, la integración de distintas disciplinas artísticas en una misma obra, el concepto se desarrolló plenamente alrededor de 1900, albergando un sentido más profundo. A partir de entonces, la creación de una obra bajo estos ideales también toma en consideración el poder transformador del arte en el ser humano y la sociedad, como actor en la elevación poética de la existencia en todas sus dimensiones. En la obra de Behrens y, especialmente, su casa de 1901 queda patente el deseo de desarrollar una estética total e integradora que conduzca, además, a la reconciliación entre el arte y la vida (Bryant, 1997, pp.57-60)³.

\section{La Colonia de artistas de Darmstadt}

En 1899 Ernst Ludwig, Gran Duque de Hesse, inauguró la Colonia de artistas de Darmstadt (Darmstädter Künstlerkolonie), siendo esta parte de las exhibiciones enmarcadas en las reformas de las artes aplicadas durante el fin de siglo (Windsor, pp.14-21). Múnich, que era el gran centro artístico del momento ${ }^{4}$, sirvió de inspiración al Duque en sus deseos de convertir Darmstadt en un nuevo centro artístico mediante la promoción económica del arte y la artesanía. Según él mismo, promover la cultura artística aumentaría el poder de la nación, motivo por el que la reforma de las artes debía comenzar entre los elementos básicos que forman parte de la vida cotidiana de una sociedad, es decir, la artesanía (Koch y Fuchs, 1901, p.25). En el programa de la exposición se hace evidente esta defensa heredada de la actividad artesanal en detrimento del trabajo industrial, lo que

por sí mismos, además de la importancia que presenta la estilización rítmica y la descomposición geométrica del espacio en las superficies (Banz y Beierdorf, 2015, p.6).

${ }^{3}$ Gabrielle Bryant interpreta de forma completa en un artículo de 1997 para Cuaderno de notas este concepto en la obra de Peter Behrens (Bryant, 1997, pp.57-76).

${ }^{4}$ En 1892 tuvo lugar en la ciudad la fundación de la primera secesión alemana, la Secesión de Múnich (Münchener Secession). Esta asociación, compuesta por un total de 79 artistas, tenía como objetivo transformar la posición social del artista, alejándolo de los cánones académicos y demandando nuevos espacios de exposición en los que el artista se enfrentase de forma directa con el público (Chodzinski, 2007, pp.116-124). 
cambiaría por completo cinco años más tarde en la III Exposición de Artes y Oficios de Alemania en Dresde (1906): “El artesano debe ser guiado a través de una educación y modelos artísticos: un trabajo personal, en el que la inteligencia, el gusto, las ideas propias y el espíritu más tradicional posible lleguen a expresarse. Esto no lo puede hacer la máquina" (Koch y Fuchs, 1901, p.25) 5 .

Para llevar a cabo el proyecto, el Duque contó con siete artistas que durante dos años debían diseñar viviendas completamente equipadas que ejemplificasen en sí mismas la reforma de las artes (Windsor, 1981, pp.14-21). El arquitecto principal de la Colonia fue Joseph Maria Olbrich, quien diseñó la casa del Duque. Además, ocho ateliers se dedicaron a exposiciones colectivas, entre otras instalaciones como restaurante o floristerías, que fueron construidas de forma temporal para la exposición (Koch y Fuchs, 1901, pp.20-30). Con motivo del evento, también se editó un catálogo escrito por el dramaturgo Georg Fuchs y Alexander Koch (Koch y Fuchs, 1901), junto con un escritohomenaje titulado "Un documento de arte alemán", que fue realizado por Behrens e impreso en su primero diseño tipográfico, la Behrens-Schrift ${ }^{6}$ (Behrens, 1901a).

\section{La Haus Behrens}

Behrens actuó en la Colonia como arquitecto autodidacta, dado que su formación se había limitado a la pintura y hasta entonces solo había diseñado para los Talleres Unidos de Múnich algunas piezas de joyería y vajilla, entre otros objetos (Windsor, 1981, p.11). Su vivienda fue acompañada de un pequeño libro titulado Haus Peter Behrens, en el que desarrolló los fundamentos en los que basó su diseño e incluyó los planos del edificio junto con los datos de los fabricantes encargados del mobiliario (Behrens, 1901b). Las únicas exigencias a las que debió atender Behrens a la hora de proyectar la casa fueron que ocupase en planta el menor tamaño posible y se adaptase a una familia con una cantidad media de miembros (Behrens, 1901b, p.7).

Formalmente, la vivienda se expresa como obra de arte total debido a la integración en la misma de otros trabajos y objetos diseñados por Behrens, que participan minuciosamente de la misma estética. No obstante, la esencia de esta idea subyace del mismo modo en la concepción espacial de la casa, desde el planteamiento de la planta y el alzado, incluyendo el jardín y pasando por el diseño de cada pieza del mobiliario, en el que cada detalle fue planificado acorde a una armonía determinada, incluso, para dar lugar a una atmósfera concreta.

\footnotetext{
${ }^{5}$ Traducción propia: "Der Handwerker muss durch künstlerische Erziehung und künstlerische Vorbilder angeleitet werden: persönliche Arbeiten, in denen Intelligenz, Geschmack, eigene Ideen, und möglichst volkstümlicher Geist zum Ausdrucke kommen, zu leisten. Das kann die Maschine nicht." (Koch y Fuchs, 1901, p.25).

${ }^{6}$ Esta fuente fue publicada en 1902 junto con otros diseños para bandas y encabezados (Behrens, 1902).
} 
Behrens comprende la vivienda como un espacio en el que distintos aspectos vitales deben ser estimulados posibilitando la convergencia de los distintos intereses de una vida propiamente cultural y elevada. Para dar lugar a esta confluencia, Behrens recurre a uno de los principios que definirían la arquitectura moderna durante el siglo XX: la interrelación de espacios. En este caso, Behrens lo denominó el "principio de posibilidad de separación”:

“El principio de la libre ‘posibilidad de separación’ para cada miembro individual de la casa planteaba el cometido de alcanzar las mejores condiciones para cada habitación en la medida de lo posible. Esto había de suceder a través de un sistema no intencionado de ordenamiento derivado del uso pretendido de la interrelación de los espacios en todas las dimensiones." (Behrens, 1901b, p.8) ${ }^{7}$.

Así, la libertad de circulación a través de las habitaciones de la planta baja de la vivienda se basó en un principio de coexistencia que no renunciaba a la privacidad de cada uno de los integrantes de la familia [1]. El espacio quedó proporcionado de forma que no existiese ninguna habitación mayor orientada al uso común, pudiendo utilizar las puertas correderas para abrir o cerrar el espacio a conveniencia (Behrens, 1901b, p.8). El ejemplo más ilustrativo de esta correlación se encuentra en la primera planta, entre la sala de música, concebida como sala de fiestas, y el comedor. Ambas habitaciones se encuentran conectadas por grandes puertas correderas, que enlazan al mismo tiempo la sala de música con el vestíbulo por el otro lado [2]. Entre estas habitaciones existe, además, un juego de contrastes gracias a los colores que proporcionan los materiales utilizados en cada espacio, así como gracias al desnivel de dos escalones de la sala de música para favorecer su uso como sala de fiestas (Behrens, 1901b, p.8).

\footnotetext{
${ }^{7}$ Traducción propia: "Das [...] Prinzip freier Separierungs-Möglichkeit für jedes einzelne Mitglied des Hausstandes stellte [...] die Aufgabe, für jeden Raum nach Thunlichkeit die größten Verhältnisse zu gewinnen. Das mußte geschehen durch ein ungezwungen aus den Zweckbestimmungen abgeleitetes System des In- und Aneinanderordnens der Räume in allen Dimensionen“"(Behrens, 1901b, p.8).
} 


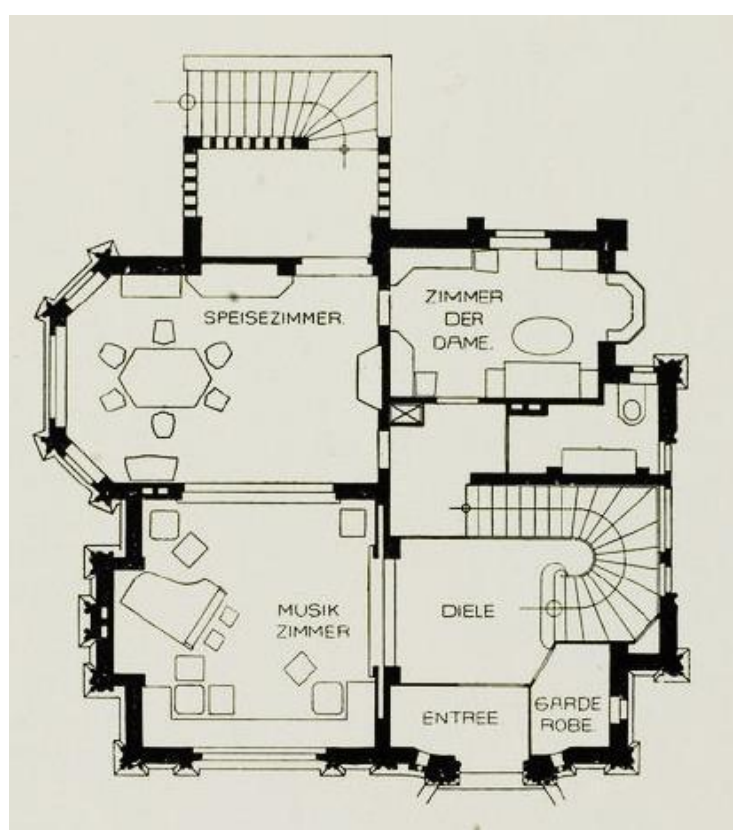

1. Planta baja de la Casa Behrens. Abajo a la izquierda la sala de música, conectada al vestíbulo (dcha.) y al comedor (arriba). Fuente: (Koch y Fuchs, 1901, p.333)

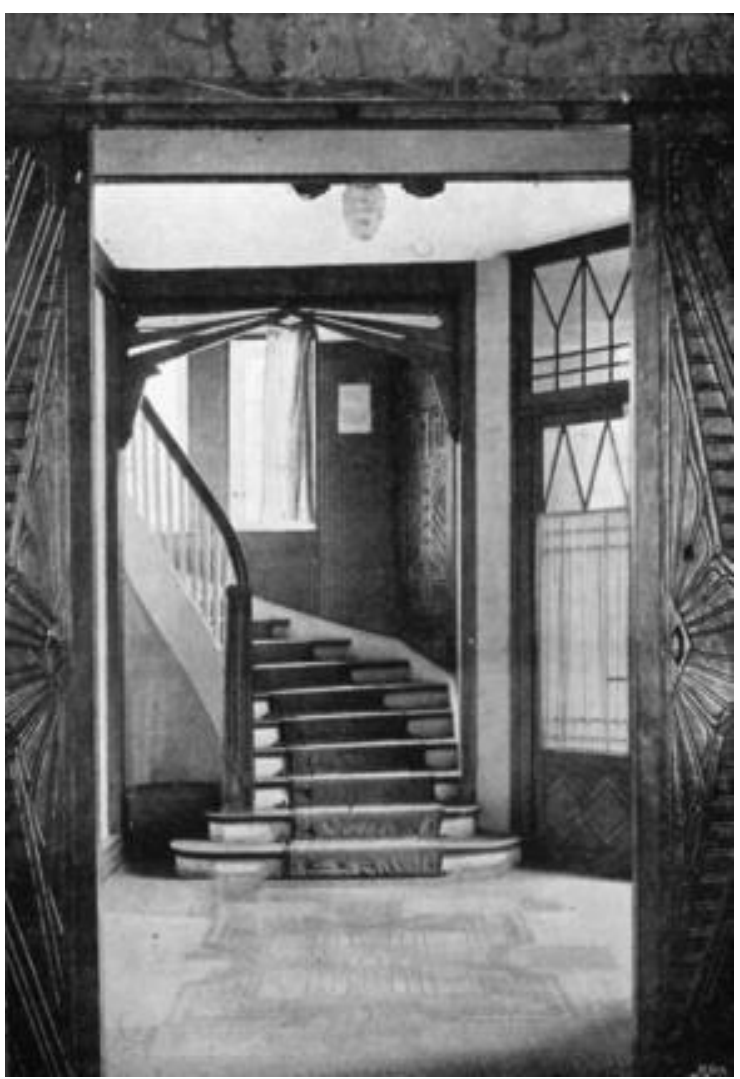

2. Vista del vestíbulo y las escaleras desde la sala de música, a través de las puertas correderas. Fuente: (Koch y Fuchs, 1901, p.363) 
El aspecto de la sala de música fue intenso debido a la decoración con marquetería y taracea en madera oscura. Su mobiliario se compuso de sillones de brazo, sillas y bancos de abedul barnizados en negro. El techo fue revestido de dorado, mientras que las paredes se decoraron con mármol rojo y amarillo. Al fondo de la estancia, un gran nicho albergaba el cuadro Un sueño, realizado por Behrens en $1897^{8}$ [3]. El comedor, por su parte, era una habitación de muebles blancos con lámparas de plata y cristal, bastante iluminada gracias a las puertas acristaladas que daban al jardín (Windsor, 1981, pp.2431) [4].

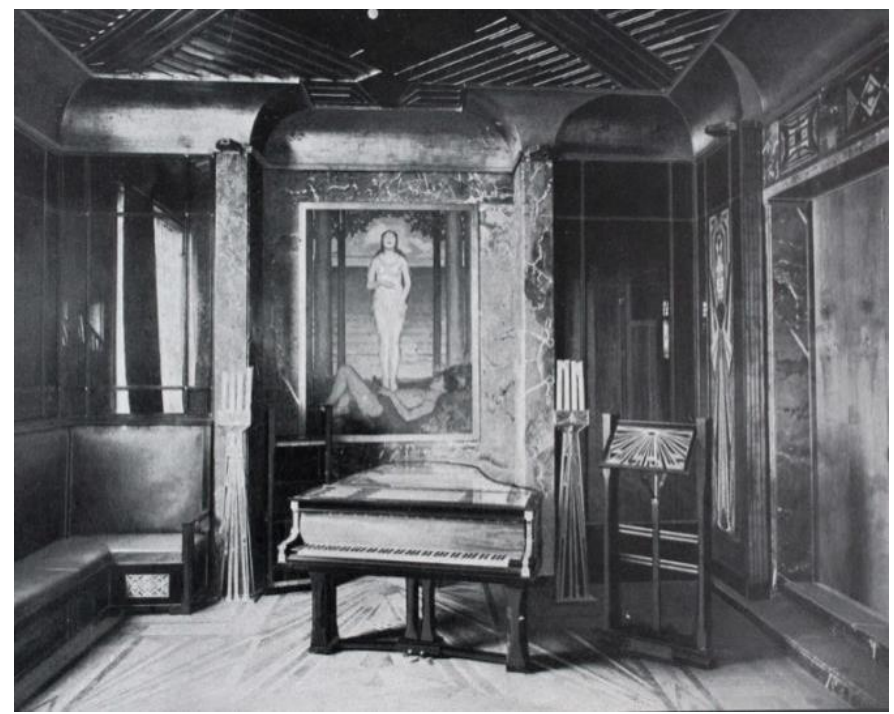

3. Sala de música de la Casa Behrens. A la derecha, las puertas correderas que accedían al comedor. Fuente: (Koch y Fuchs, 1901, p.366)

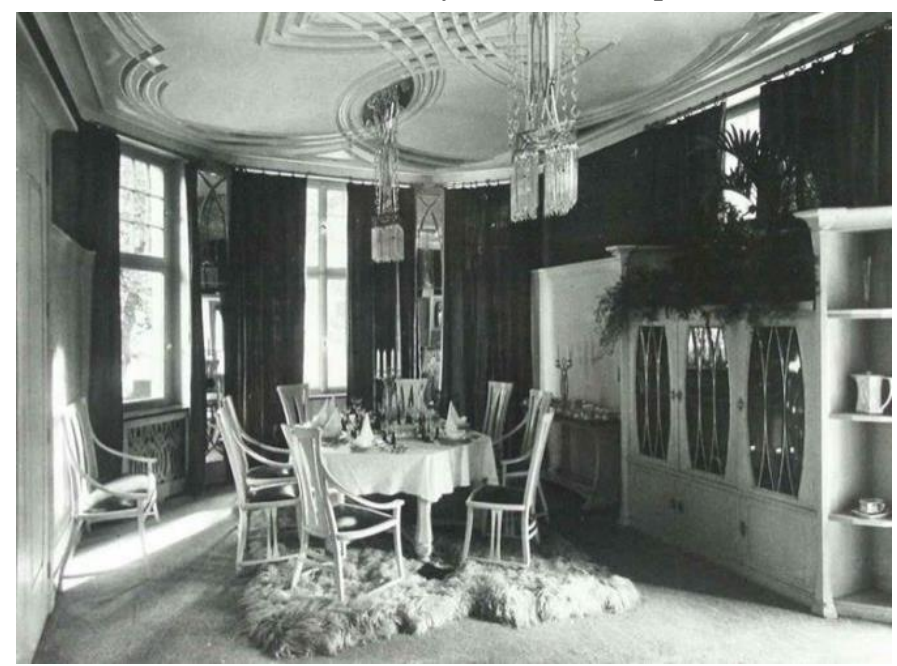

4. Comedor de la Casa Behrens. En el techo se observa el ornamento de tipo lineal, que se repite en las sillas, los espejos y la cubertería. Fuente: https://www.pinterest.de/pin/333266441147431492/ (Consulta 20/12/2017)

\footnotetext{
${ }^{8}$ Es posible experimentar una recreación más o menos exacta del interior de la Casa Behrens gracias al proyecto de recreación virtual del Dr. Jörg R. J. Schirra y Kai Buchholz (BUCHHOLZ, Kai y SCHIRRA, Jörg, 2000-2006).
} 
En la segunda planta, aunque en menor medida, las habitaciones también se encuentran conectadas [5]. Sobre el comedor se halla el dormitorio de la señora, que se comunica con el de la hija a través de un cortinaje. Lo mismo ocurre en la habitación de trabajo del señor, que ocupa la superficie equivalente a la sala de música en la primera planta. Esta y la biblioteca son un mismo espacio que puede separarse mediante una gran tela. Al otro lado de la planta se sitúa el dormitorio del señor, comunicado con el de la señora a través de una puerta. En el ático se dispone la habitación de invitados, que cuenta con su propio baño y balcón, con el objetivo de facilitar su independencia del resto del núcleo familiar (Behrens, 1901b, p.9).

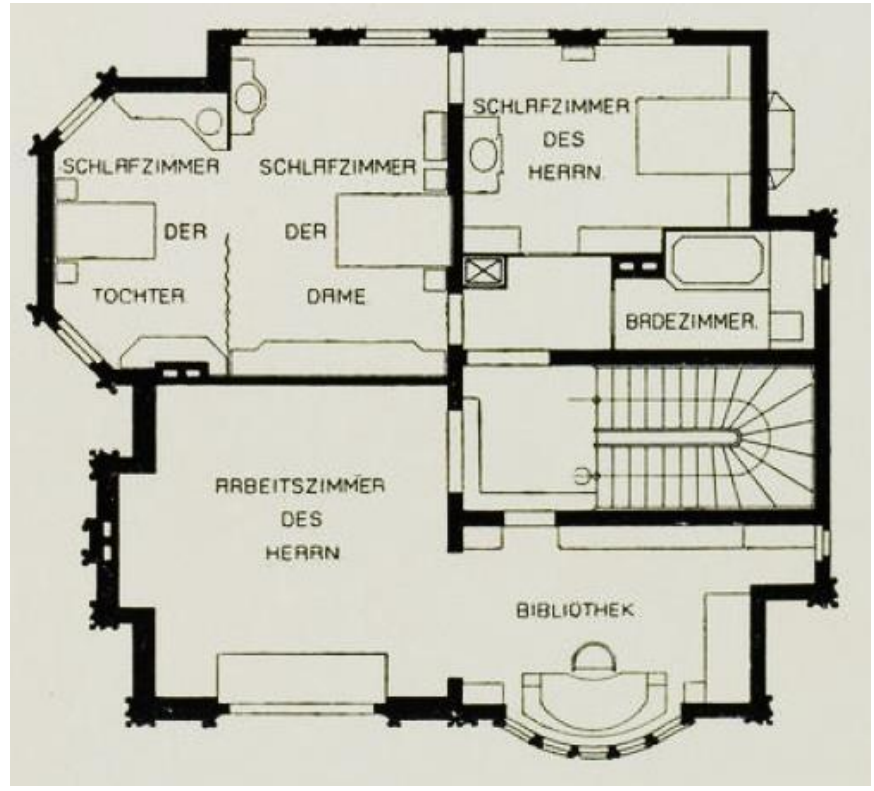

5. Segunda planta de la Casa Behrens. Arriba a la izquierda el dormitorio de la señora, conectado al de la hija por un cortinaje (izda.), y al del señor por una puerta (dcha.) Abajo, el atelier del señor (izda.), unido a la biblioteca (dcha.). Fuente: (Koch y Fuchs, 1901, p.333)

En esta serie de correlaciones entre los distintos espacios resulta fundamental la integración del jardín, ya que proyecta el espacio habitable de la vivienda hacia el exterior. Este, que incluía un parque infantil, un banco y una fuente, se diseñó también de acuerdo con un "sistema de ejes", un segundo principio que debía proporcionar un efecto de amplitud dentro de la vivienda, gracias a la percepción visual que relaciona los distintos espacios con el exterior, tal y como explica Behrens (Behrens, 1901b, p.10):

"Si se sigue con el ojo, desde el nicho del piano de la sala de música, el eje, que a través del ancho de la sala de música conduce por el rellano y la escalera hasta la ventana de la escalera en la pared oeste, se abarca un espacio muy extenso en proporción a la dimensión total de la casa, que, aunque se 
extienda a través de diferentes espacios, se resume a través del medio arquitectónico en una cierta unidad." 9

El sentido estético y poético de la vivienda en su conjunto no exime a Behrens de ocuparse del carácter funcional de la misma, exhibiendo con ello algunos rasgos de la funcionalidad propios de la arquitectura moderna. El sentido práctico a través del que escogió los materiales para su vivienda también queda expresado por el propio Behrens en su libro al justificar, por ejemplo, la utilización de un aislamiento de corcho en el techo de la habitación sur del ático con el objetivo de protegerla contra el frío y el calor, así como en el sótano, para asegurar una temperatura equilibrada. Para conseguir una buena acústica en la sala de música, su suelo fue realizado con mosaicos de madera y moqueta, y las paredes fueron recubiertas de cristal azul. El comedor también está pensado para guardar el frío en verano, motivo por el que su suelo se compone de mosaicos de teselas, pero al mismo tiempo incluso para proporcionar calor en invierno, razón por la que la cocina se encuentra justamente dispuesta a modo de sótano bajo esta habitación (Behrens, 1901b, p.12).

\section{Conclusiones}

La habilidad de Behrens para conjugar de forma tan temprana lo estético y lo práctico bajo los ideales de la obra de arte total convierte la Casa Behrens en una obra que debe ser estudiada mucho más allá de los límites de su dimensión estética. La austeridad exterior de la vivienda, que prescinde en sus muros blancos de ornamentación, hizo que la crítica la alabase entonces, limitándose a un aspecto estético, por su modernidad y racionalidad, en comparación con el exceso decorativo del resto de edificios diseñados por Olbrich (Hoeber, 1913, p.10). Pero son, sin embargo, la concepción del espacio y la funcionalidad los elementos que Behrens destaca de forma prioritaria en su libro al fundamentar el diseño de su propia obra.

Tal y como apunta Gabrielle Bryant, el principio de la obra de arte total fue algo que pareció acompañar a Behrens durante toda su carrera, haciéndose especialmente evidente en su etapa en Düsseldorf (1903-1905), sus edificios para la exposición de Oldenburg en 1905, la fábrica Hoechst en Frankfurt am Main (1920-1924) y su trabajo como diseñador industrial, gráfico y, con todo ello, corporativo para A.E.G (Bryant, 1997).

\footnotetext{
${ }^{9}$ Traducción propia: „Wenn man von der Klavier-Nische des Musikzimmers aus mit dem Auge der Achse folgt, welche durch die Breite des Musik-Zimmers über den Vorplatz und die Treppe bis zum Treppenfenster in der West-Mauer führt, so umspannt man einen im Verhältnis zu den Gesammtmaaßen des Hauses sehr umfangreichen Raumkomplex, der, obwohl er durch verschiedene Räume sich erstreckt, doch durch architecktonische Mittel zu einer gewissen Einheit zusammengefaßt wird.“ (Behrens, 1901b, p.10)
} 
Hay que tomar en consideración que resulta precisamente la creación de la identidad corporativa, y con ello la proclamación efectiva de la unión entre la industria y el arte, la que hace asegurar a la autora que esa es "seguramente la formulación más nueva e importante de la concepción de la 'obra de arte total' en los albores del siglo XX" (Bryant, 1997, p.70). Esta es la paradoja de un artista universal, que supo adecuar la idea de obra de arte total, surgida bajo la influencia de aquellos que luchaban contra la industrialización, a la verdadera revolución total del arte, aquella que supo ver el arte como agente configurador de la vida y la industria moderna.

\section{Referencias bibliográficas}

BANZ, Claudia y BEIERSDORF, Leonie (2015), Jugendstil. Die große Utopie, [exposición celebrada en el Museo de Artes y Oficios de Hamburgo, del 17-X-2015 al 7-II-2016], Museum für Kunst und Gewerbe Hamburg, Hamburgo.

BEHRENS, Peter (1902), Behrens, Schriften, Initialen und Schmuck nach Zeichnungen von Professor Behrens, Rudhard'sche Gießerei, Offenbach am Main.

BEHRENS, Peter (1901a), Ein Dokument Deutscher Kunst, Verlag der Verlags-Anstalt F. Bruckmann A-G, Múnich.

BEHRENS, Peter (1901b), Haus Peter Behrens, ed. Herbert, Darmstadt.

BRYANT, Gabrielle (1997), "Peter Behrens y el problema de la obra de arte total en los albores del siglo XX”, Cuaderno de notas, n 5, pp. 57-76.

BUCHHOLZ, Kai y SCHIRRA, Jörg (2000-2006), [reconstrucción virtual de la Casa Behrens]. http://jrjs.bplaced.net/www/Work/Projects/Behrens/index2.html (Consulta 20/12/2017).

CHODZINSKI, Armin (2007), Kunst und Wirtschaft - Peter Behrens, Emil Rathenau und der dm-drogerie markt, Kulturverlag Kadmos, Berlin.

DEWIEL, Lydia (2002), Schnellkurs Jugendstil, ed. DuMont, Colonia.

HOEBER, Fritz (1913), Peter Behrens, Georg Müller und Eugen Rentsch, Múnich.

KADATZ, Hans-Joachim (1977), Peter Behrens Architekt-Maler-Grafiker und Formgestalter 18681940, VEB E. A. Seemann Verlag, Leipzig. 
KOCH, Alexander y FUCHS, Georg (1901), Grossherzog Ernst Ludwig und die Ausstellung der Künstler-Kolonie in Darmstadt von Mai bis Oktober 1901, ed. Koch, Darmstadt.

NERDINGER, Winfried (dir.) (2007), 100 años de Arquitectura y Diseño en Alemania Deutscher Werkbund 1907-2007, [exposición celebrada en el Museo de Arquitectura de la Universidad Técnica de Múnich, del 19-IV-2007 al 26-IX-2007], ed. Prestel, Múnich.

WICHMANN, Siegfried (1984), Jugendstil Floral Funktional, Bayerischen Nationalmuseum, München.

WINDSOR, Alan (1981), Peter Behrens Architect and Designer 1868-1940, The Architectural Press, London. 